Johannes Andreas STISSER

De Machinis Fumiductoriis curiosis sive Fumum

Impellendi Intra Corpus

Hamburg 1686 

D. $0 . M, I$.

JOHANNIS ANDREÆ STISSERI. D.

Medici Hamburgenfis.

De

MACHINIS

GUMIDUCTORIIS

CURIOSIS

five

Fumum Impellendi Intra Corpus

INSTR UMENTIS

Eoruinguc in praxi medicâ adbibendi

ratione of ufu

EPISTO L A

Ad

Illuftrifimos Viros

MAGNAE SOCIETATIS REGIE

ANGLICAN N.

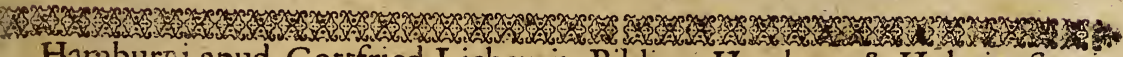
Harnburgi apud Gotrfried Liebezeic Bibliop. Hamburg \& Holmienf ANNO M DC LXXXVI, 


\section{Hippocrates de Arte.}

\section{Mivero invenire aliquid eorum qux inventa nondum funt, aut femi perfecta ad finem perducere fcientia votum ac opus effe videtur.}




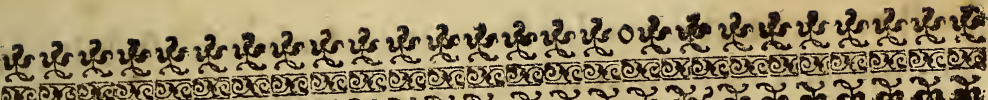

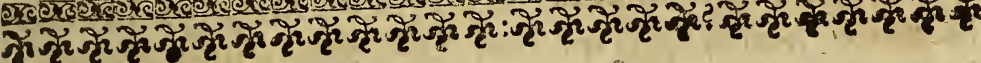

\section{O. M. I.}

Magnæe Socictati

Regix.

\section{Anglicana}

S. $P$.

7) Ulta \& egregia extitifle no-

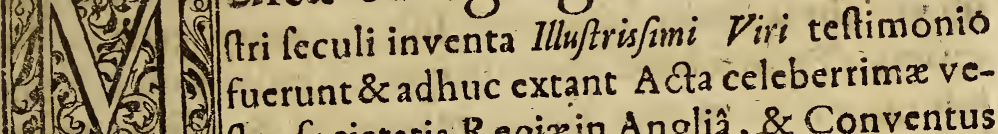
Itr $x$ focietatis Regixin Angliâ, \& Conventus non Academix curiofx in Germaniâ : Quorum omnium, utiles progreffus in hunc us que diem crefcere cernimus. Nemohinc inficias ibit prefentis feculi induftriam \& curiofa, myfteriorum natura fcrutinia cum indefefsâ experimentandi cupiditate cunctis preteritis palmam praripere. Ut plerosque his in rebus excellentes eruditoque orbi notos (quorum encomia ftante Oceano vix peribunt)prateream, confiderentmodo velim, âClariffimo veftre terre virorum pari Bacone Verulámio \& Roberto Boylaxo adbuc fu- 
perftite, venerabilis veftrx focietatis membro fpectabili, in Philofophiâ experimentali, àClariflimo Malpigio autem \& Stenone, non Ánglis quidem laude tamen non minoribus in Anatorniâ tancunincrementi factum, quantum in præteritis feculis fibi nunquam promittere audebit antiquitas. Nec negabit moitalium quisquam nifi fupidi ingenij de fanguinis circulatione doctrinam (egregium pariter hujus feculiinventum) ad rationalem morborum medelam haud parumattuliffe lucis; contra quam licet acerrime pugnaverint fcholarum fectatores, invicta tamen manfit veritas à modernis medicis immortalitatis Ratux infculpta. Quales Chymia fecerit progreflus, non eft quod fufius oftendam, loquuntur hâc dere pariter Clariffimorum Virorumi cripta ub:vis obvia firmis admodum fulcita experimentis. Et quamvis Pyrotechnia olores in eam ab omni tempore in. huncusque diem fuperciliosè invexerint $\&$ adhuc invehant, ignorantiæillorum ignofcendum erit \& flocci faciendum hominum judicium, qui causâ ne quidem cognitâ inique judicant. Præbet hujus rei teftimonium hominum. falus ex Chymicis gratis fub parva doli petita, quam $\mathrm{Ga}_{\mathrm{a}}$ lenica medendimethodus innumeris decoctis naufeabundis A pozernatibus, electuariis, aliisque ejusdem farine medicaminibus fub magnà quamvis dof comparare haud poterat. Non eft ut oltendam Chymicas analyfes ad rerum naturalium fcientiam captandam pra reliquis omnibus facere: Cedunt his prifci fubtilitatum magiftri inutiles ; \& 
ut infinita Clarifimorum virörum circa hujus rei verita. tem teftimonia præteream, audiamus unum faltem illum, perignem Philofophun Helmontium. Laudo inquit benignum mibi Deum qui me in Pyrotecloniami vocavit etc. Siquidem Chymia principia babet non logismis parta, fed qux per naturam funt cognita of per ignem confpicua, preparatǵ, intellectum ad penetranda occulta naturce, ponitque invejtigationem in naturam ulteriorem, quam alise fcientice ommes fimul of penetrat usque ad ultimas profunditates veritatis realis, quia operatorem intromittit ad. primas radices illarum rerum cum dearticulatione operationum natisre vo poceftatum artis, cumque maturatione virtutum feminalis um Ec. Sed ne nimis diu in laudandis modernorim, medicorum præclarè inventis inmoremur (cum præfertim pagellæ noftræ laude digna illorum inventa recenfere non fufficiant) cuncta jam recenfita ad medelam graviffimorum morborum haud parum feciffe in propatulo eft. Cui \& non parum attulerunt opis à Medicis \& chirurgis celebrioribus inventa inflrumenta non exigui numeri, quo. rum pleraque ita comparata ut quicquid corpori noxium exterminent, quod vero huic falubre per varia naturxe munctoria aut vias arte factas introducant. Horum in. numerum omni jure recepta ef Regneri de Graaff medici Delphenfis clariffimi machina pro clyfteribus injiciendis. cujus figuram \& ufum videre licet in tractatu ejus de cly. fteribus. Hoc autem infrumentum in praxi medicà ufus habuit egregios, cujus beneficio unicuique commodum. A 3

fuit 
fuit fibimet ipfi injicere clyfmata, quorum ufum antea refpuebant fapius quos fingularis quadam tenebat verecundia. Non minoris utilitatis exiltit machina ea ratione? confecta ut tabaci aliorumá; fumus in anum immitti queat à Barbaris aut Turcis ut volunt primò inventa, qui fi. fulas profuctione fumi tabaci ex corio pararunt flexiles, ne frangerentur $a b$ externà injuriâ, quemadmodum $a b$ illis vel ob leveen caufam obfervare licet, quas ex terrâ limosâ albicante in variis Europa locis parare folent nobis vocat3 Jobads Pfeifien.

Coriace is autem fintulis non folum pro attractione \& luctione tabaciBarbari ufi funt, verum etiam pro fumo tabaci in anum immittendo uti coperunt doloribus colicis \& torminibus alvi favientibus, ita ut fiftul Lubverfa partem oridicatam in anum immitterent, alteram vero partem capitellum fcilicet ex figulinâ terrâ paratum, inflarent, quo pacto quilibet fibimet ipf fumum ingerere potuit. Hoc inventum ex eoq; redundantem utilitatem cum cernerent curiofi quidam veftræA A gliæ pro commodiori inflatione \& minus nauleabunda aliam excogitarunt machinam, nimirumut longius diflarent ab ore folia illa tabaci accenla \& fumus adverfantibus minorem crearet moleftiam, pars infuperori dicata in a num intromitti minime opus haberet. Cujus notitia miror quód plures medicor um hactenus latuerit, cum rarius unum alterumve offenderim qui ejus ufum \& Aructuram habuerit cogniiam, quamvis machina usilitatem incomparabilem didicerim ut poltmodum oftendàm. 
dam. Ultrum de eâaliquid̀ typis mandatum fit nefcio; vix tamen credo,cum hactenus neque ipfemet follicite in quirens viderim nec offenderim, qui de eâ aliquid excufum viderit. Qux rationes me moverunt ut hanc machinam àMedicis quibusdă clariffimis tacitè habitam in lucem ederê, flimulque adjungerem figuras \& Anglican \& noftreinventionis infrumenti, ab Anglicano quidem ron multum externè, plurimum vero interne differentis, cum al tero pro inflatione fumi commodius fit \& infpiffata quoque balfamica in fumum refoluta introducat quax Anglicani inftrumenti foramina omni procul dubio obtararent: His non iniquum duxiadjungere quidnam utile ab iis in praxi obfervaverim. Structura autem hujus machinx externa inferius vergens non, multum differt ab inftrumento R. de Graaff,funiculum autem arbitor interne non tam exacta conclufione opus habere quemadmodum R. de Graaff de fuo tradit, ita tamen debere praparari ut inflatus nullo modo äer ad latera ejus tran. feat, extremaque ejus pyxidi fuperius, cannulax autem inferius, ur firmiter fint adnexa opus eff. Interim parspyxidis interior laminâ ferreá obducitur, ne pyxis ex ebore aut ligno confecta à calore diffiliat t. Eadem lamina ferrea operculi partem interiorem claudit, relicto foraminulo pro infpiratione, quemadmodum ex figuris videre licet quz majoris lucis ergo ad caicem adjuncta funt. In pyxidis parte inferiori ad bafin lamina perforata notatur in Anglicano infrumento cujus loco in noftro pileum ferreum margine perforato 
foratỏ adaptavimus cum placentâ ferreâ qux ignienda \& fundo pilei feu capitello potius immittenda venit, ne obtu: rentur foramina \& fumi tranfitus prapediatur, influmentum quoque igne parietibus internis proxime accedente non nimis incalefcat, quemadmodum in Anglicano notavi, injectis prunis aur globulo ferreo candente indito, additis fimul profuffitu fpeciebus. Et tali modo conffructa machina, cannula quæ in reliquis clyfteribus ordinaria oleo aut $æ a b o$ oblinita in anum immittitur, placenta ferrea candenspilei capitello imponitur, adjiciuntur fpecies pro fuffitu, clauditur operculum cujus tibiam inflat $æ$ ger, \& fic fumus abs qve molefia intra corpus abit. Hoc inftrumento in praxi diverfi mo. dèufusfum, inditis pro diverfitate morborum variis pro fuffiturebus, Primo aistem Barbarorum Anglorumque morem fecutus, adjectis profuffitu tabaci foliis egregia præftitiin colicis doloribus ubi à morbo rejectis remediis carminantibus, anodynis, difcutientibus, tandem ad hujus inftrumenti ufum veluti ad facram anchoram confugere ra. tus fum, non fine laudabili fuc ceffu, cum dolores \& cruciatus intolerabiles fumo vix ingreffo incantamenti inftar fedari a: nimadverterem in plurimis, nec aliter in me ipfo, qui cums ante annum \& quod excurrit à graviffim is alvi torminibus divexarer, omnibus fruftra tentatis, tantum commodi ex machinx ufu percepi, ut cum circa noctem tabaci fumum inflarem, poftridie circa meridiem lurgens omnis doloris expers ad mea iterum redierim negotia, meosque agrotantes vifi-

taverim 
taverim. Neý; minorem opentulife didecin hyftric is affectionibus \& doloribuisnephriticisł ne ipsâ (quarnvis in hâc non femper exoptatus notetur eventus) in Epilepfià, A poplexiâ aliisớ; loporofis affe Ctibus egregie paroxyfmos difcutit, fedopus eft adftans famulus vel ancilla ut inflandoxgrivicesagat, quippe in hoc deficiente facultate. Et ita adultis aque ac infantibusopitulatur, quorum epilepfram vermin ofam ex fententià inde folutam vidi, rejectis paulo poft inflationem pervomitum \& feceffrim lumbricis. Jnhydrope (praprimis vero tym panitide difficilioris curationis ) interdum commodo non vacat, nempe flatus inclufos quafi refolvit, ferofam colluviem exficcat, \& fegnem naturam excretionis officii admonet. In Dyfenteriâ, Tenefmo, Fluxu albo mulierum, exficcando vifcidam materiami relolvendo, tonum partium reftituendo egregia, fecit, necminori virtute egit in menfium luppreffiune, foetu mortuo: \& fecundinis expellendis, in ipfo quoque partu difficili promovendo. Neque contemnendam ex eovtilitatem perceperunt herniâ flatulentâ divexati, cum flatusinteftinorum refolvat $\&$ tonum partium membranofarum relaxatum humores exficcando reftituat finulg; roboret. Hxc cum obfervaffem à foliis Tabaci quibus ob fal volatile (quod penetrantiffimum in (e haber)egregia vis ineft refolvendi, penetrandi,exficcandi,cum forti quadam difcuffione, alia quoque pro luffitu experiri confultum ratus, extractá ex oleis fotidis \& refinis balfamicis preparavifale volatilifcatentia, ad- 
ditis variis cáloris ope in fumum facile refolubilibus, quorum mixtura fuffirienta extitere admirandæ virtutis, quibus in graviffimis \& chronicis quoque affectionibus feliciffme perDei gratiam ufusfum, \& proftant in teftimonium hujus rei apud nostrochilci quidam pro fumo, in gravillimis \& defperatis ferè doloribus admirand $x$ virtutis. Sed objicient quildam, valvulam coli impedire omnem verfus fuperiora afcenfum, quemadmodum in anatomicis à $\measuredangle \psi_{i}^{\prime}$ fem per docuit, inftituto inflationis verfus fuperiora experimento; idcirco fumum non pertingere polfe ad tenuiorá inteftina \& ventriculum, quorum flatulentæ diftenfiones tamen doloris colici caufa citraipfius coli paffionem exifunt. His autem non, femel contrarium oftendere potuiffem fi adfuiffent mihimedicinam facienti colico morbo decumbentibus, quibuspeculiaris quidam liquor cum decocto ceparum proclyftere injectus paulo poft per os iterum rediit, quemadmodum ex odure \& colore adftantes, \& 2 gri ipfi ex fapore, minus grato licet, manifefte potuerunt colligere. Obfervavimus hoc iplum nón lemel à liquore noftro, quo raro ufifunus in colicà, quod non moverit vomitus, non tamen liquore iplo per os femper rejecto, nifi in graviffmis \& defperatis alvi tormi . nibus, quibus mirê opem attulit internis \& externis cunctis fruftra tentatis. Internon paucos veriffimus hujus reiteftis haud adeo pridem fuit Reverendus quidam \& eruditione clarifimus Vir, cui ruedicinam in talicafu fecimus, adflante chirurgo, meo confilio liquorem injiciente, quem paulopont aliqua 
- aliqua ex parte fuperius ten diffe ex vomitu rejectis non fine ftupore notarunt ager \& chirurgus, mihique paulo poft advenienti monftrarunt. Sed citraliquoris noftri additionem non abfimile quid à clyfmate injecto faotum medici clariffimi memorix mandarunt, inter illos âtem praprim is $\mathrm{Cla}_{2}$ riff. Th.Kerkringius, cujus egregia in Spicilegio fuo anato-

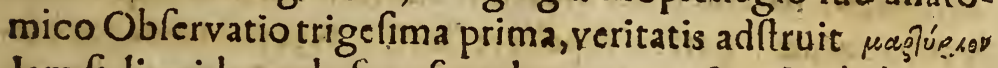
Jam fi liquida mole fua afcendere queant \& valvula limites transgredi: quid dubitabimus in fumum refoluta inflationis adminiculo lubtilitate \& levitate atomorum fuorum facilius poffe petere eandem viam \& quidem minori negotio? Huc usqueigitur (ut ad propofitum redeam) in praxi pervenerenoftrx machinx experimenta, cujus funiculi aut fumiductus conficiendi rationem hactenus experiri haud potui, cum datâ licet operâneminem offenderim coriarium in florentiffimâ noftrà urbe, qui canaliculum cjusmodi flexilê feu fumiductũex corio parare fciverit Ang licano fimilem, quamvis in fuperioribuis Germanja locis parari audiverim tales, guales ex Angliâ quondam accepi. Curabo itaque inpofterum aliunde adferri funiculos, donec in noftris locis parari queant, quibus accommodari poteft pyxisnoftrx inventionis, lococboris ex folido ligno conficienda \& interius lamina ferrea cuftodienda ne rumpatur: Inferiori parti cannula in anum immittenda facili negotio adjungitur, transverfim tamen pro commodiori ingreffu \& liberiori fumiexitu. It aque fine magnofumptu haberi poterit machina cunctis fere 
conmoda \& utilis, qux aphdadultos \& infantes ufunon vacat. Sed diobus jam recenfitis non incenfultum arbitror, ut tertium addam fumiductum aliúm quendam ex ferroparandum, cujus ftructura â fintulis noftris tabacifugis parumb differt, nifiut capitellum cláudi poffir operculo, cujustibia inflata per foramen ori dicatum fumum emittat, quem ad varias corporis partes impellere licet. Necelfe tamen, eft femoto operculo, ut inditze pecies accendantur more folito ab ignis flammâ per attractionem oris \& fuctione quadam; quo facto clauditur operculum \& inflatur ejustibia, tunc fumus ad foramen atractorium tenditinverfum \& fatis valide expellitur: Quem fumum fi intra corpus pellere lubet adjungere licet foramini atrractorio fiftulam aliam rectam aut inflexam variis corporis meatibus inferendo aptam. Hocinfrumentum excogitavimus quondam in cafu defperato pro Empyematico, cujus incifum latus per turundam quotidic incredibilem puris fundebat copiam; inflato tamen fumo ex Nicotianà Scabiosâ, Hederâ terreftri, Tuffilaginç, Myrrhâ \& fimilibus, beneficio noftrifumiductus, non folum brevi difparuit foctor: fed \& imminutuseft fluxus, cum exoptaco denique prater fpem integrafanitatis fucceffu, non - omiffis tamen aliis ad curam fpectantibus, Haud adeo pridem in fimiliferecafu Nicotianæ fumus per Tabaci fiftulam inverfam inflatus (cum fumiductu noftro deftitueremur) non contemnenda nobis praftitit, Nec commodo vacat per hoc inftrumentum inflatus fumus auribus in tinnitu coper.

rum, 
rum, gravi auditu. Oculis in fluxione illorum, pforâpalpebrarum, aliisq́; ophtalmicis morbis; Naribusin hyftericâ paffione, Epilepfrâ A poplexiâ, electis ratione diverfitatis morborum diveris \& convenientibus pro fuffitu remediis. Reliqua cum machinis noftris tentanda, viris in arte verfatis relinquimus, haud dubitantes, quin ex illarum ufu non, contemnendam utilitatem capturifint. Et fic advosredicrunt Viri llluftris/imi qua veftra fuiflenemo inficias ibit, melioritamen facie donata \& experimentis haud contemnendisaucta effe, judicabic candor vefter \& religuuseruditus orbis. Quamlicet faciem \& veftra jam opera elegantiorem acceperit rudis ille \& fordidus barbariæ foetus, inventis celebrioribus quoque aliquid utile addi poffe unice arbitror ad fcientiarum omnium augmentum facere, Accipite ergo Viri llluftrisfimi veft a noftris juncta, tribuite hoc affectui crga Excelientisfimam Veftram Societatem quen hodie omnis teltatur eruditorum nunerus, \& quamvisindiesad Vos ve. niant noftris ele gantiora, non tamen diffido \& noftros quo. que conatus à Vobis probari, ut defint vires. Quod ubi audicro, habebounde gaudeam. Hisitaq; Valete.

\section{B 3}

Tabú 


\section{Tabula Prima:}

1 Nftrumentum â Barbaris inventum profuctione fumi tabaci, cujus A. Capitellumex terrâfigúlinâ uftum pro foliorum immiffione qux ąccendi debent : Huic nectitur B. funiculusfeu fumi ductus ex corio flexilis unâ fuâ extremitate; alterâverò C. Cannulx lignex pro fuctione fumi adhretet.

\section{Tabula Secunda.}

A Inftrumentum Anglicanum in fundoha$A_{+}$bens B. Craterem live laminam perforatam. C. Funiculus flexilis ex corio, filo cupreo circumvolutus cum admota D. Cannula.

\section{Tabula Tertia.}

A. Inftrumenti noftrx inventionis pyxis eA. burnea aut lignea, cujus interior pars laminâ ferreâ obducta. B. Pileus ferreus, cujus in marginè confpiciuntur foramina pro fumi

tran- 
tranfitu; Profunditatifeu patinx qux margini firmiter adhærent, inditur. C. placenta, ferreaignienda \& adjiciuntur fuffimenta: deinde clauditur pyxis operculo. D. cujus medium penetrat. E, tibia. F. Capitellum habens pro inflatione ori admovendum. G. Pars inferior pyxidis, per cujus foramen defcendit fumusin $\mathrm{H}$. funiculum flexilem ex corio $\mathrm{pa}$ ratum, \& pervenit tandem ad I, cannulam in anum immittendam, cujus $K$ inferior pars foraminahabetpro fúmi exitu.

\section{Tabula Quarta.}

Figura. I.

A Pyxis claufacum immiffo pileo. B. canA + nula. C. funiculus ex corio cavus, cujus, $D$. extremitas unajungitur $E$. foramini pyxidis inferiori. F. altera verò. G. cannulx foraminulo.

Figu- 


\section{Figura. 2.}

A Fiftula fumiductoria ferrea tabacalem A.fiftulam xmulans, cujus B. capitello cavo, induntur fpecies pro fuffitu qux igni admotx per C. foramen ori dicatum accenduntur, deinde clauditur capitellum D. Operculo cujus E. tibia inflatur \& pellitur fumus per foramen ori dicatum. Aut fi intra corpus impellere animus eft, adjungitur. $\mathrm{F}$. Cannula pro commodiori immiffione. G. G. G. fiftula fumiductoria conftructa.

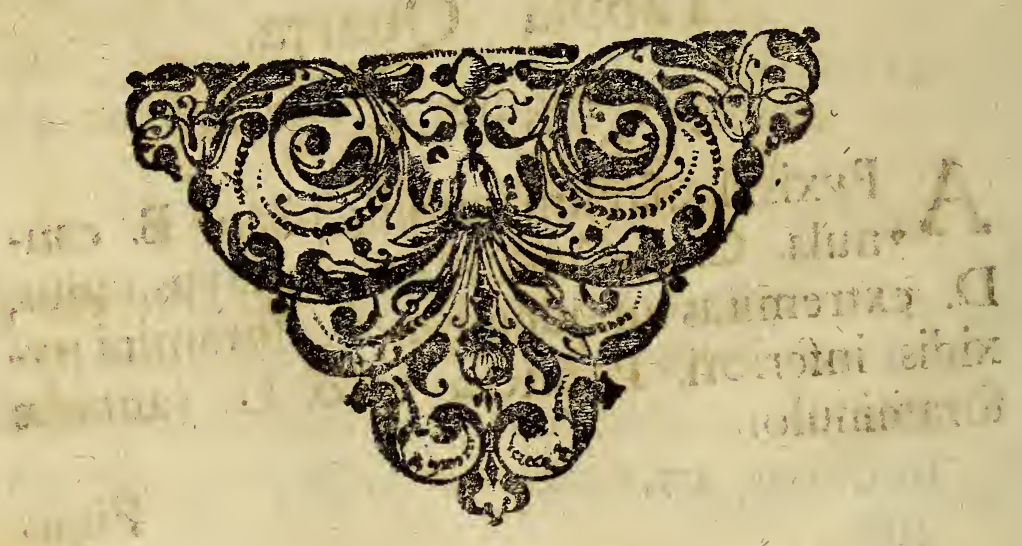





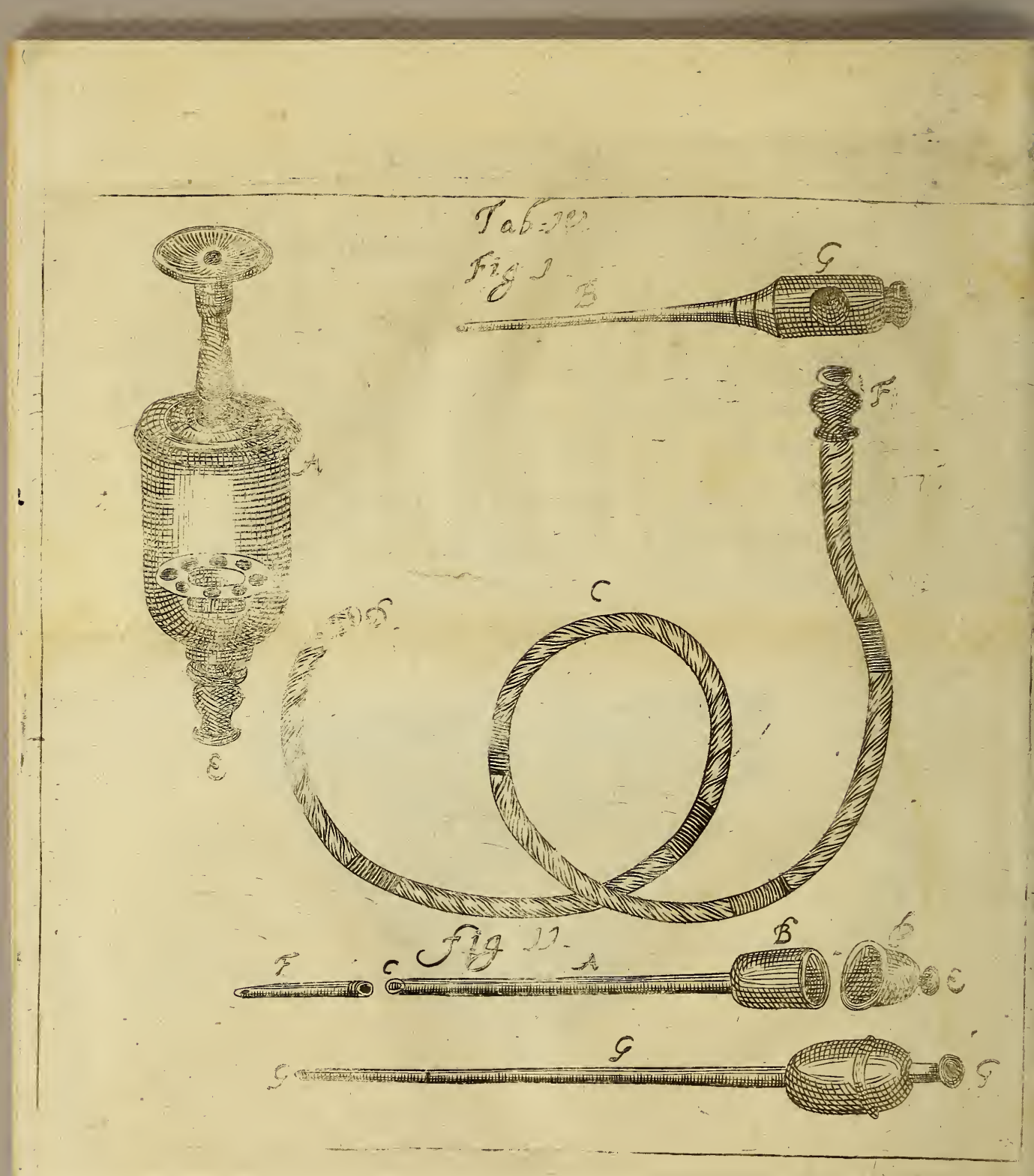




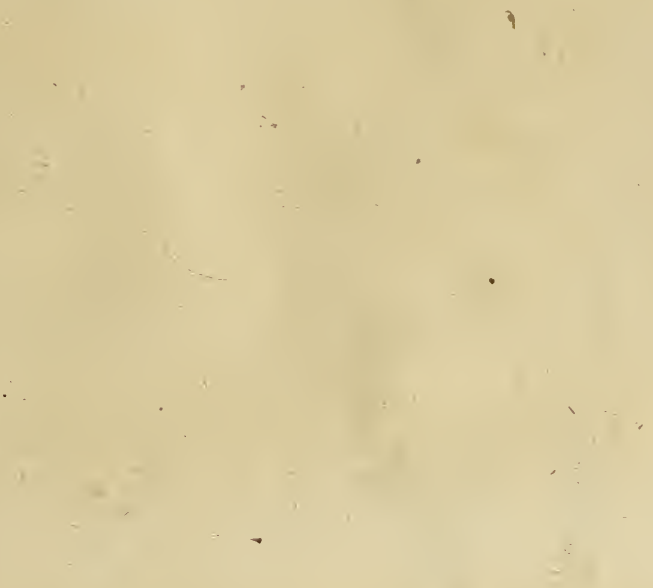




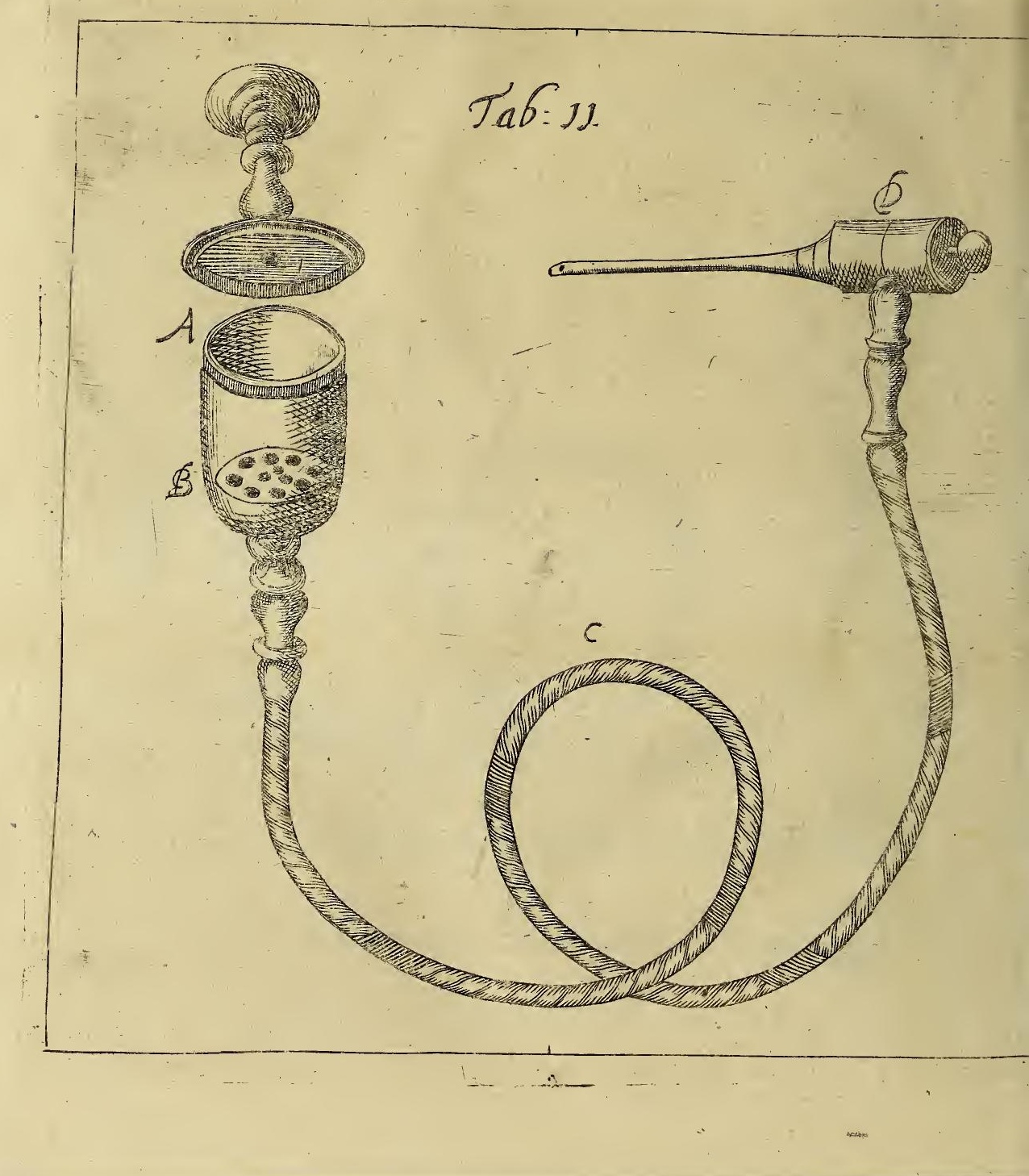





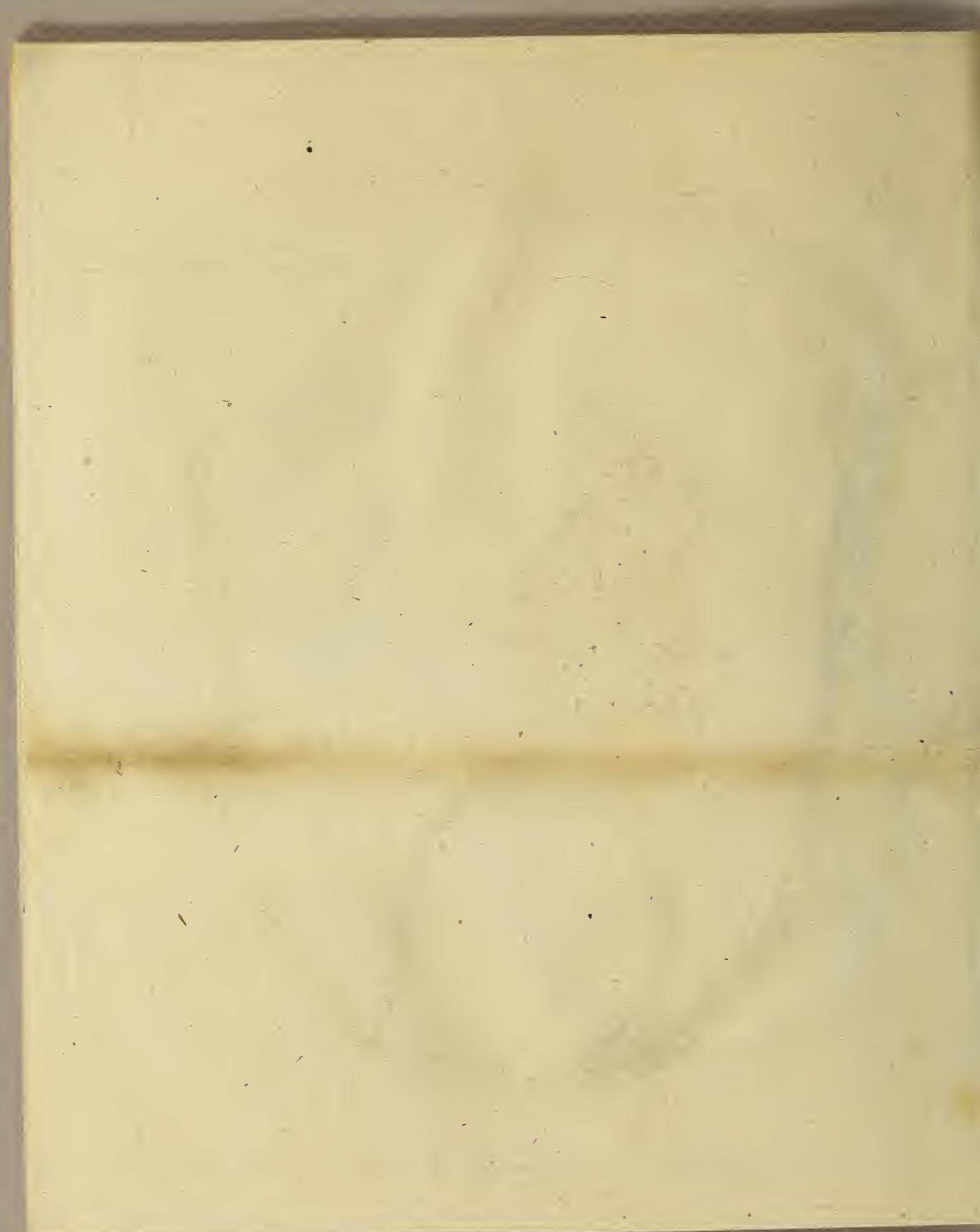


J68 6

- S8cid

,

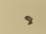

$\because$

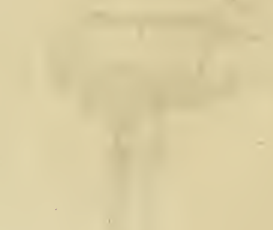

$-$

.
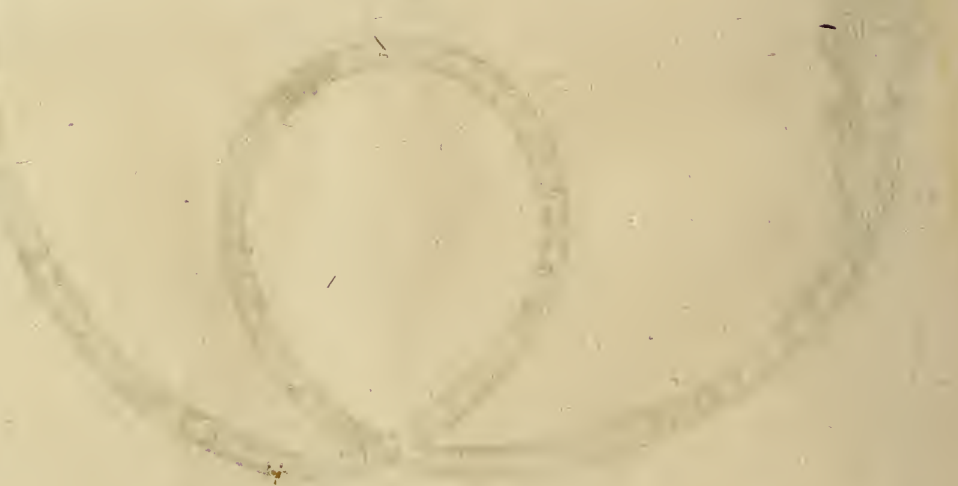
